\title{
Atopic Dermatitis Host and Environment Model: Revisiting Therapeutic Options
}

\author{
${ }^{1}$ Immunoallergology Department, Hospital de Santa Maria, Centro Hospitalar Lisboa Norte (CHLN), Lisbon, Portugal \\ ${ }^{2}$ Immunoallergology Department, Hospital Professor Doutor Fernando Fonseca, Amadora, Portugal \\ ${ }^{3}$ Immunoallergology Department, Centro Hospitalar de Trás-os-Montes e Alto Douro, Vila Real, Portugal \\ ${ }^{4}$ Allergy Unit, Pedro Hispano Hospital, Matosinhos, Portugal \\ ${ }^{5}$ Basic and Clinical Immunology Laboratory, Medical School, Porto University, Portugal
}

\section{KeY WorDS}

atopic dermatitis; therapeutics;

immunologic factors; environment;

quality of life

\author{
Corresponding author \\ Cristina Lopes \\ Faculdade de Medicina \\ da Universidade do Porto \\ 4200-319, Porto, Portugal \\ Phone: +351225513600 \\ E-mail: cristina.lopes.abreu@gmail.com
}

Doi

10.23822/EurAnnACI.1764-1489.125

\begin{abstract}
Summary
Atopic Dermatitis affects both children and adults and is a serious health concern in many countries. $A D$ is a complex disease with host and environmental factors underlying its pathology. Its treatment is multidimensional reflecting the diverse nature of its triggers and includes emollients, topical steroids and calcineurin inhibitors among others. Immunological dysfunction can be addressed broadly with systemic immunosupressors and specifically with monoclonal antibodies. Dupilumab, which targets IL-4 and IL-13 was granted approval for treatment of moderate-to-severe AD. Biologics targeting IgE/Th2 pathways may have its role in patients with overlapping $A D$ and asthma.

Psychological distress can exacerbate symptoms and is associated with increased severity of $A D$. Environmental triggers, such as, allergens can be addressed in selected cases with allergic immunotherapy.

In this paper, we discuss $A D$ treatment and propose a new step-by-step approach aiming at maintaining disease control and improving quality of life.
\end{abstract}

\section{Introduction}

Atopic disorders represent a global health problem with a number of studies demonstrating an increase in the prevalence of asthma, allergic rhinitis (AR) and atopic dermatitis (AD) over the last four decades (1). Although current estimates point to $\mathrm{AD}$ cases leveling off or even decreasing in some countries, such as, the United Kingdom and New Zealand, AD remains a serious health concern in many countries, particularly in the developing world where the disease is still very much on the rise (2). The sharp increase in allergic diseases between the early 60s and the late $80 \mathrm{~s}$ is perceived to be a consequence of an intense migration from rural to urban regions, and from poor, developing countries to more affluent, heavily industrialized regions of Europe, Asia and the Americas. The recent biodiversity hypothesis on allergic diseases (3) claims that not only the loss of macrodiversity determined by climate change and pollution is associated with adverse health effects, but also that the loss of microdiversi- ty is associated with various inflammatory conditions, including asthma and allergic diseases. As such, a fundamental role for microorganisms in human health, whether indigenous or environmental, is becoming increasingly evident.

Besides the importance of the environment in the development of allergic diseases, an increased familiar predisposition for the development of these conditions may exist. This observation led researchers to hypothesize that host genetic factors could be involved in the pathogenesis of $\mathrm{AD}$. The description, back in 2006, that loss-of-function mutations in the filaggrin (FLG) gene were a strong genetic risk factor for $\mathrm{AD}$, became a significant breakthrough regarding prognosis and treatment. FLG monomers aggregate keratin filaments into tight bundles, resulting in the collapse and flattening of corneocytes that maintain both skin barrier integrity and normal stratum corneum (SC) lipids. Therefore, mutations in the FLG gene may increase skin permeability, predisposing individuals to skin allergen penetration and subsequent infection. These mutations have also 
been correlated with other atopic disorders such as atopic asthma, although with conflicting and less clear results (4).

Dysfunction of innate and adaptive immune responses are typical features of AD. Atopic skin exhibits decreased levels of antimicrobial peptides and a decreased number of dendritic cells when compared with the skin of patients with other inflammatory skin diseases. $\mathrm{AD}$ patients have increased risk of developing rhinitis and asthma, which suggests a systemic Th2 allergic predisposition in this population (5).

We can consider that host and environmental factors contribute to $\mathrm{AD}$ pathogenesis and manifestations. The former includes genetic background, namely filaggrin gene mutations, innate and adaptive immunological dysfunction and psychological aspects that interfere with patient's quality of life. Environmental factors include allergens and skin microbiome that can modulate expression and severity of $\mathrm{AD}$ (figure $\mathbf{1}$ ).

The treatment of patients with $\mathrm{AD}$ is therefore multidimensional aiming at restoring skin hydration and lipid defects, downregulation of allergen-driven skin inflammation, elimination of skin pathological inhabitants, and addressing the pruritus that perpetuates the vicious cycle of scratching. Recently, new immunomodulators have emerged as complementary treatment strategies to conventional $\mathrm{AD}$ therapies, because these molecules not only diminish symptoms but also address immunological dysfunction (6).

Our aim is to provide an updated revision on the treatment options for $\mathrm{AD}$ that target both the host (skin barrier, immunological deviation) and the environmental factors (allergens and skin microbiome) underlying this pathology, with special emphasis given to new immunomodulatory drugs.

\section{Host factors}

\section{Skin barrier}

The first approach to symptoms management is therapy directed at skin barrier impairment (7). The aim should be to maintain skin care, improve skin repair, and keep a healthy skin barrier, in order to suppress the inflammatory response and keep itching under control (7).

Figure 1 - Atopic Dermatitis multicomponents model. AD has a complex pathogenesis with multiple players. Innate and adaptive immune dysfunction promote Th2 and Th1 driven inflammation and changes in the normal skin microbiome. The microbiome dysbiosis potentiate the irritating action of allergens, air pollutants and smoke. Immunological factors also act on resolution and skin repair leading to chronic lesions characterized by lichenification and fibrosis. The genetic background can, in some subjects, be responsible for the skin barrier impairment leading to a more severe disease. Intensity and extension of lesions are the main determinants of symptoms (pruritus, pain, skin discomfort). Psychological factors such as anxiety can potentiate symptoms and symptoms can lead to psychological distress such as depression and quality of life impairment. Adapted from Anderson (58).

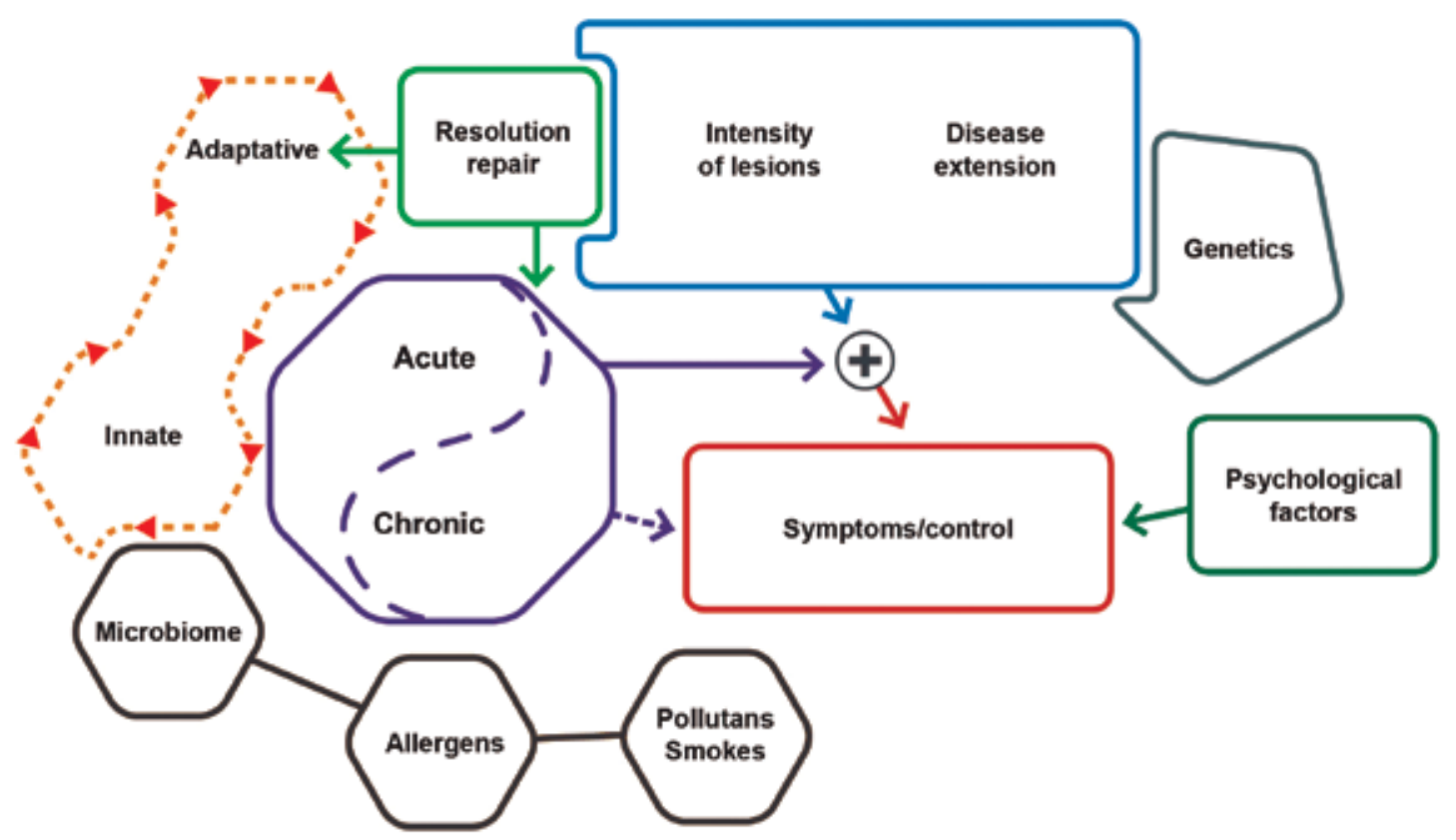


A. Lopes, A. Sokolova, C. Abreu, C. Lopes

Emollients are the first step in the treatment regimen of $\mathrm{AD}$ because they promote skin care and repair, restore epidermal function, suppress inflammation and maintain itch control (8, 9). Emollients are topical preparations and can be delivered via a variety of formulations, including creams, ointments, oils, gels, and lotions. Emollients are normally used in a liberal way, aiming at maintaining minimal xerosis (8). Their use may be especially relevant in patients with FLG deficiency since this leads to defects in the formation of the stratum corneum (SC), decreases the ability to maintain its hydration, and induces a parallel elevation in $\mathrm{pH}$, lipid bilayer disorganization, percutaneous allergen exposure and xerosis (10).

Emollients are designed to maintain the skin's softness and hydration and can be occlusive, humetant or lipidic. Occlusive emollients maintain the external hydrophobic layer of the skin surface reducing transepidermal water loss levels (TEWL); humetant emollients have hydrophilic hydroxil groups and are capable of retaining water within the skin, either by attracting water from the dermis or from the external environment (when relative ambient humidity is greater than $70 \%$ ); lipidic emollients, such as ceramides, replenish the lipid component of the $\mathrm{SC}$, which is decreased in $\mathrm{AD}$, and by doing so, they improve transepidermal water content in children (11).

Emollients have numerous beneficial effects for $\mathrm{AD}$ patients including decreasing the number and increasing the time to flares and reducing the amount of topical corticosteroids needed (12) Randomized controlled trials have demonstrated the benefits of long-term use of emollients in xerosis control, which translates into better quality of life (QoL) of patients (13). When the regular use of emollients fails to achieve satisfactory skin care and reduced symptoms, other topical therapies are required(8).

Topical corticosteroids (TCS) are the core of anti-inflammatory therapy, being used in children and in adult patients when the lesions fail to respond to good skin care and regular use of moisturizers alone (9). They act on a multitude of immune cell populations, namely $\mathrm{T}$ lymphocytes, monocytes, macrophages, and dendritic cells, decreasing the release of pro-inflammatory cytokines (8). TCS also reduce Staphylococcus aureus bacterial load, likely via decreasing the inflammatory cytokines that inhibit antimicrobial peptide production.

TCS are utilized for active inflammatory flares of disease and for prevention of relapses, decreasing both acute and chronic signs of $\mathrm{AD}$, as well as pruritus (8). A meta-analysis of randomized controlled trials has advocated a proactive approach of maintenance therapy for those patients with repeated outbreaks at the same body sites. When used once to twice weekly at these particular body locations, TCS reduced the rates of relapse and increased time to first flare relative to the use of moisturizers only. TCS are grouped into classes according to anti-inflammatory potency, and selection of steroid should be guided by location, extent and acute or chronic nature of skin lesions, pa- tients' age, and disease severity. Low-potency TCS are indicated for mild disease, flexural and facial skin lesions, young children and pregnant women. High potency TCS are preferred for older patients, lichenified and chronic prurigo-like lesions and palms (14).

It has been shown that TCS have a greater absorption rate and systemic uptake in patients with clinically severe disease, when compared to patients with mild or moderate disease, suggesting caution in their use in more advanced stages of the disease and in infants (15). The incidence of reported side effects from TCS use is low; however, most studies fail to follow patients long-term for potential complications. Cutaneous side effects include purpura, telangiectasias, striae, focal hypertrichosis, and acneiform or rosacea-like eruptions. Of greatest concern is skin atrophy, which can be induced by any TCS, though higher potency agents, occlusion, use on thinner skin, and older patient age increase this risk. Continuous application of TCS for long periods of time should be avoided, to limit the occurrence of negative changes. Proactive, once to twice weekly application of mid-potency TCS for up to 40 weeks has not demonstrated these adverse events in clinical trials (8).

Topical calcineurin inhibitors (TCIs) are a class of anti-inflammatory topical therapy that inhibits calcineurin-dependent T-cell activation, decreasing the production of inflammatory cytokines (8). Pimecrolimus and tacrolimus are approved in the EU from 2 years of age and above. TCIs can be used in patients who fail to respond to other topical therapeutics such as TCS or as a complementary approach $(8,9)$. The long-term use of TCIs is supported by robust data, documenting safety and efficacy, while data supporting long-term TCS use are limited to lowto mid-potency products (8). Despite this, a meta-analysis by Broeders et al demonstrated that TCIs and TCS led to a similar percentage of patients presenting improvements in dermatitis and of treatment success rates both in children and adults (16). Pruritus in $\mathrm{AD}$ is multifactorial depending on other mediators than histamine like nerve growth factor, substance P, protease, and cytokines/chemokines (thymic stromal lymphopoietin (TSLP), IL-2, IL-4, IL-13, and IL-31) (17) explaining with anti-histamines have demonstrated little utility despite their frequent use topical anti-histamines, because of risk of sensitization, are contra-indicated (8). Oral sedative $\mathrm{H} 1$ antihistamines are not recommended because of the risk of adverse reactions such as increased somnolence or restlessness, confusion, etc. A summary of the main conclusions regarding skin barrier is presented on box 1.

\section{Immune deviation}

Systemic immunomodulatory therapy is reserved for patients with poor response to non-pharmacological or topical treatment, with persistence of symptoms and impairment of QoL (18). All 


\section{Box 1 Summary of the main conclusions regarding skin barrier.}

- The use of emollients prevents exacerbations

- Flares should be treated with topical corticosteroids

- Topical calcineurin inhibitors should be used as a complementary approach, especially in sensitive skin areas

immunomodulatory agents should be adjusted to the minimal effective dose once response is achieved, and topical treatments should also be maintained in order to allow the lowest dose and duration of systemic agents. Both non-specific and specific immune systemic therapies are available for these patients.

Non-biologic systemic drugs used for adult AD include cyclosporine, corticosteroids, azathioprine, methotrexate (MTX) and mycophenolate mofetil (MMF), which exert their immunosuppressive effects by reducing inflammatory cell numbers and pro-inflammatory cytokines expression (19). Phototherapy is also frequently used as a second-line treatment for moderate-to-severe $\mathrm{AD}$ in adults (20).

Cyclosporine is an immunomodulatory drug that inhibits interleukin IL-2 and T-lymphocytes. According to Consensus-based European guidelines for treatment of atopic dermatitis it is the first choice for systemic treatment of severe adult $\mathrm{AD}$ patients who are unresponsive to topical therapy and require systemic immunosuppressive treatment (21). An initial daily dose of 2.5$3.5 \mathrm{mg} / \mathrm{kg} /$ day and a maximal daily dose of $5 \mathrm{mg} / \mathrm{kg} /$ day, divided upon two single doses, is recommended. A dose reduction of $0.5-1.0 \mathrm{mg} / \mathrm{kg} /$ day every 2 weeks is desirable as indicated by clinical efficacy. It can be used as a continuous therapy, but a maximum duration of 1-2 years has been suggested to avoid adverse events such as nephrotoxicity, hypertension, tremors, headaches, paresthesia, nausea, diarrhea, myalgias, electrolyte imbalance, hyperlipidemia, hypertrichosis and gingival hyperplasia. Patients receiving cyclosporine should be monitored for blood pressure and renal parameters, as cyclosporine is known to induce structural and organic kidney damage. Nephrotoxic effects are more likely to occur if the daily dose exceeds $5 \mathrm{mg}$ $\mathrm{kg}$ body weight, serum creatinin values are elevated or elderly patients are treated (22). Cyclosporine may be used 'off label' in children and adolescent patients showing a refractory or severe course of disease (23).

Systemic corticosteroids decrease the transcription of several mediators involved in the pathogenesis of $\mathrm{AD}$, including cytokines, chemokines and adhesion molecules, by binding to regulatory elements on many genes, thus leading to resolution of inflammation (19, 24). Despite rapidly improving disease activity, systemic corticosteroids (oral or parenteral) have a largely unfavorable risk/benefit ratio for adult $\mathrm{AD}$ treatment $(19,24)$ and long-term use is not recommended $(18,19,24)$. Also, a rebound flare and increased disease severity is frequently seen after discontinuation of systemic steroids. Short-term (up to 1 week) treatment may be an option to treat acute flares in exceptional and severe cases of $\operatorname{AD}(19,21,24)$.

Azathioprine is a purine analog that inhibits DNA production and reduces leukocyte proliferation thus decreasing inflammation (18). It is used off-label for the treatment of severe $\mathrm{AD}$ in adults, in particular in the UK and USA $(18,19,24)$. It may be used off-label when cyclosporine is either not effective or contraindicated (21). Although several studies have demonstrated QoL improvement and symptomatic control with azathioprine usage in $\mathrm{AD}(18)$, data on efficacy and safety are still sparse. Adverse events of azathioprine include gastrointestinal disturbances, liver dysfunction and leukopenia $(19,24)$.

Methotrexate (MTX) is an antimetabolite that regulates the immune system and inflammatory processes, by interfering with folic acid metabolism through blocking of RNA, DNA and purines' synthesis (18). Several studies suggested that MTX is well-tolerated and effective in the treatment of moderate-to-severe forms of $\mathrm{AD}(19)$ even if its use is off-label. Nonetheless, liver and bone marrow toxicity have to be monitored before and during MTX therapy. The adverse events most commonly causing discontinuation of MTX treatment include nausea, fatigue, hepatotoxicity, hematological abnormalities, pulmonary toxicity and drug interaction. Folic acid supplementation is recommended during treatment with MTX to reduce the likelihood of hematologic and gastrointestinal toxicity.

Mycophenolate mofetil (MMF) is also an antimetabolite that blocks the purine biosynthesis pathway selectively inhibiting Band T-cell proliferation. Several case reports and small studies showed its efficacy when used off-label in adult patients with $\mathrm{AD}$ who were unresponsive to cyclosporine therapy $(19,21)$. The main adverse events reported during MMF therapy were nausea, fatigue, flu-like syndrome and liver enzyme alteration.

Phototherapy with artificial UV radiation is frequently used as a second-line treatment for moderate-to-severe $\mathrm{AD}$ in adults (20). Narrowband UVB is preferred over broadband UVB for AD treatment if available (9). UV irradiation is able to modulate the immune response of $\mathrm{AD}$ patients through upregulation of FoxP3-positive regulatory $\mathrm{T}$ cells, whose number is directly correlated with the degree of AD severity score. Phototherapy can be used as short- and/or long-term treatment. TCS and emollients can be associated with phototherapy to reduce flare-ups, whereas TCIs should be avoided to limit the risk of carcinogenesis $(9,20)$. Phototherapy must be performed conscientiously, especially in children, and must take into account the patient's features and overall condition (20).

Severe refractory $\mathrm{AD}$ patients that fail to improve with systemic immunosuppressive therapy, or those who experienced import- 
A. Lopes, A. Sokolova, C. Abreu, C. Lopes

ant side effects, may benefit from biologic therapy. Biological therapies for $\mathrm{AD}$ include several monoclonal antibodies, of which omalizumab and dupilumab are the best studied. Currently, dupilumab is the only biological therapy approved for treatment of moderate-to-severe AD by the Food and Drug Administration (FDA) and European Medicines Agency (EMA).

Omalizumab is a humanized monoclonal antibody that binds to the high-affinity $\operatorname{IgE}$ receptor, preventing $\operatorname{IgE}$ from binding to the surface of several cell types including mast cells, basophils, dendritic cells and eosinophils, and so blocking mastcell degranulation and decreasing the release of cytokines and recruitment of other inflammatory cells (25). Treatment with Omalizumab is currently indicated in adults, adolescents and children ( $>6$ years of age) with severe persistent allergic asthma and in refractory chronic spontaneous urticaria (26). Although data from case series and case reports documented clinical benefit of $\mathrm{AD}$, some studies showed no improvement of disease with Omalizumab both in adults and children $(27,28)$. Nevertheless, a recent randomized clinical trial found that Omalizumab significantly reduced atopic dermatitis severity and improved quality of life in a pediatric population (4-19 years old) with atopy and severe $\mathrm{AD}$ despite highly elevated total IgE levels at baseline (29). Due to $A D$ heterogeneity, it seems that some patients are most likely to respond to anti-IgE therapy: lack of filaggrin mutations and lower elevations of total serum $\operatorname{IgE}$ are factors associated with a likely favorable response to Omalizumab (30, 31). Based on case reports and case series, targeting IgE seems to be an option in patients who have overlapping allergic diseases such as asthma (32). However at this time, available scientific evidence does not support its use for the treatment of AD (21) and larger RCTs are needed.

Dupilumab is a fully human monoclonal antibody that targets IL-4R $\alpha$ and inhibits signaling of IL-4 and IL-13, both of which are key Th2 cytokines that play an important role in AD.(33) The data supporting its efficacy and safety came from two randomized, placebo-controlled, phase 3 trials, SOLO 1 and SOLO 2, involving 671 and 708 adult patients, respectively, > 18 years of age with moderate-to-severe AD (34). Dupilumab has a favorable safety profile with no dose-limiting toxicity and few adverse events, including nasopharyngitis, upper respiratory tract infections, conjunctivitis, headache, injection-site reaction and back pain $(33,34)$. Dupilumab, is indicated for the treatment of moderate-to-severe $\mathrm{AD}$ in adolescent and adult patients who are candidates for systemic therapy $(35,36)$. European Guidelines for the treatment of $\mathrm{AD}$ recommend dupilumab as a disease-modifying drug for patients with moderate-to-severe $\mathrm{AD}$, combined with daily emollients (21). Dupilumab has also recently been approved for treatment of severe asthma (37) and severe chronic rhinosinusitis with nasal polyps (38). Box 2 summarizes the main conclusions regarding non-specific and specific immune systemic therapies.

\section{Box 2 Main conclusions regarding non-specific and specific immune systemic therapies.}

- Systemic therapy should only be used if topical therapy fails

- Cyclosporine is the first-line option for patients who require systemic immunosuppressive treatment

- Systemic corticosteroids should only be used in exacerbations and for short periods of time

- Dupilumab, which targets IL-4 and IL-13 is approved for treatment of moderate-to-severe $A D$.

- Biologics targeting IgE/Th2 pathways may have its role in patients with overlapping $A D$ and asthma.

\section{Psychotherapy}

$\mathrm{AD}$ is associated with other allergic conditions and psychosocial disorders. Specifically, the prevalence of depression, anxiety and other psychiatric disorders are higher in $\mathrm{AD}$ patients than in the general population, due to social isolation, sleep deprivation and persistency of symptoms (39).

Psychotherapy through cognitive behavioral stress management has a positive impact in the burden of disease, namely on the improvement of endocrine and psychological stress responses (39). Some studies demonstrated an effective decrease of anxiety in adults, as well as in children (39). Moreover, psychological interventions are associated with better managing of symptoms and a decrease in itching intensity (39). A summary of the main conclusions regarding psychotherapy is presented on box 3 .

\section{Environmental factors}

\section{Allergens}

Historically, the relationship between exposure to allergens, specifically inhaled allergens (horse dander, ragweed pollen, timothy grass) and $\mathrm{AD}$ was demonstrated in 1918 (40). Currently, it is known that in some phenotypes of $\mathrm{AD}$ there is an immune response to allergens, mediated by IgE and T cells (41). The skin barrier function and innate immunity are involved in this pathology due to the properties of some allergens (41) that facilitate barrier disruption and cutaneous sensitization. It has been shown that exogenous protease activity of house dust

\section{Box 3 Main conclusions regarding psychotherapy.}

- Psychological distress can be an exacerbating factor of AD

- Psychological interventions may benefit $A D$ patients 
mite, insects, fungi, and pollen disrupts inter-corneocyte connections and Der $\mathrm{f} 1$ allergen disrupts epidermal tight junctions and induces inflammatory mediator release, such as IL-6, IL-8 and GM-CSF, by keratinocytes (42). Itching and delayed skin barrier recovery from mite and cockroach allergen exposure is mediated by activation of protease-activated receptor-2 (PAR2) expressed by keratinocytes and dermal unmyelinated nerve fibers (41). It is also known that PAR-2 binding capacity is enhanced by exposure to UV, with PAR-2 expression increasing in the superficial epidermis after UV exposure. Therefore, the proteolytic properties of allergens, together with UV exposure, may be a possible link behind the seasonal trend of $\mathrm{AD}$.

Despite the biological plausibility of avoidance measures, studies conducted so far provide conflicting results regarding reduced indoor contact with mite allergens (43). A recent Cochrane Review concluded that very low quality evidence was currently available regarding house dust mite reduction or avoidance measures for treating eczema (44). Several possible reasons for the failure of indoor avoidance measures exist: the effectiveness of avoidance measures is difficult to ascertain (e.g., are vacuum steam cleaning and air-filters effective?); adherence to avoidance measures is not measurable nor is the exposure to allergens outside home; and finally long-term established disease is less likely to respond to avoidance measures (43). When addressing specific immunotherapy (SIT) with aeroallergens in AD, there is conflicting evidence, with more recent literature being more in favor of it (45). SIT may have positive effects in selected, highly sensitized patients with $\mathrm{AD}$ and the best evidence so far is available for SIT with house dust mite allergens (45). There is no contraindication for performing SIT in patients with respiratory allergic diseases (allergic rhinoconjunctivitis, mild allergic bronchial asthma) and concomitant AD (22).

Regarding food allergens, the diagnosis of eczematous reactions to food requires a careful diagnostic procedure, taking into account the patient's history and sensitization patterns. The clinical relevance of sensitization often has to be proven by an oral food challenge, with the rating of the skin condition being performed by validated scores after $24 \mathrm{~h}$ and the evaluation of the eczematous reaction at a later point in time (46). Moreover, a large recent study investigating food allergy and $\mathrm{AD}$ exacerbations concluded that children with $\mathrm{AD}$ exacerbations in the absence of other allergic symptoms are unlikely to be food allergic (46). Box 4 summarizes the main conclusions regarding allergens.

\section{Skin microbiome in AD patients}

Metagenomic studies have revealed that diverse and complex microbial ecosystems inhabit the skin and are collectively known as the skin microbiome. The skin microbiome is composed mainly of members of the same four phyla that comprise the gut microbiome, although with dissimilar relative abundances. In all indi-

\section{Box 4 Main conclusions regarding allergens.}

- An immunological rationale for aeroallergen eviction exists although scientific evidence for this measure to be undertaken lacks

- Physicians should be cautious when considering food allergen eviction and only propose it after evidence of clinical relevance

- Specific allergen immunotherapy to house dust mites has shown efficacy in some studies

- Exposure to irritant environmental factors such as tobacco should be avoided

viduals, Propionibacterium species dominates in sebaceous areas such as the forehead, retroauricular crease, and back, whereas Staphylococcus and Corynebacterium species dominate in moist areas, such as the axillae. Abundant Gram-negative organisms, previously thought to colonize the skin rarely as gastrointestinal contaminants, were found in the microbiomes of dry skin habitats, such as the forearm or leg (47).

Interest in the relationship between $\mathrm{AD}$ and metagenomics is increasing. Studies show that $S$. aureus increased from $35 \%$ to $90 \%$ of the microbiome during flare-ups, with concomitant increase of $S$. epidermidis (48). It is still unclear if $S$. aureus and S. epidermidis mutually enhance each other's colonization or if S. epidermidis increase reflects an antagonistic response to an increasing $S$. aureus population. $S$. aureus produce superantigens (S. enterotoxin $A, B$ and $C$, and toxic shock syndrome toxin-1), which are important effectors in AD. They cause $S$. aureus-specific IgE production and this correlates with disease severity. Superantigens also cause nonspecific IgE production, activate $\mathrm{T}$ cells, $\mathrm{B}$ cells and macrophages, and stimulate their proliferation (49). Superantigens also induce chemokines such as CCL1 and CCL18, which bind to CLA-positive T cells in peripheral blood and thus are likely to play a role in $\mathrm{T}$ cell homing to the skin. The superantigens seem to reduce the immunosuppressive activity of certain immunosuppressive regulatory $\mathrm{T}$ cells, which may, in turn, increase inflammatory $\mathrm{T}$ cell activation (49). They are also known to induce corticosteroid resistance, thus hampering the treatment of atopic diseases.

Although infected $\mathrm{AD}$ exacerbations require specific treatment of microorganisms in combination with $\mathrm{AD}$ treatment, no evidence supports the assumption that antimicrobial treatment of colonized skin will benefit patients in the long-term (49). Moreover, combining topical antibiotic agents with corticosteroid treatment has led to no further decrease in $S$. aureus colonization compared with corticosteroid alone (50). Therefore, antibiotic treatment should be used with caution.

With the development of nanotechnology, intelligent or functional textiles with antiseptic properties are available. Such 
A. Lopes, A. Sokolova, C. Abreu, C. Lopes

textiles have been used as adjuvants and antiseptic dressings in burns and wound healing with promising results. In immunologically mediated skin diseases, and $\mathrm{AD}$ in particular, the focus has been to improve itch, severity of lesions, and skin colonization by $S$. aureus. Most of the studies of functional textiles in $\mathrm{AD}$ have investigated the use of specially treated long-sleeved shirts and pants in close contact with the skin. Cotton textiles can be functionalized with antiseptic silver salts or borage oil, which supplies unsaturated fatty acids to the skin barrier (51). Silk coated with specific antimicrobial chemical compounds and smooth ethylene vinyl alcohol (EVOH) fibers are also used to diminish physical stimuli applied to the skin (51). A systematic review provided a weak recommendation for the use of these textiles in $\mathrm{AD}$ based on low quality of evidence supporting the effectiveness of these functional textiles in alleviating symptoms and reducing disease severity (51). Nevertheless, recent studies with new biocompounds showed that chitosan-coated textiles may impact disease severity, by modulating the staphylococcal profile in the skin, and have a potential effect on QoL (52). However, further studies are needed to confirm these data, to identify which mechanisms are targeted, and to determine how functional textiles contribute to symptom improvement.

Besides pathogenic bacteria, other causes of infections in $\mathrm{AD}$ patients are virus and fungi. Herpes simplex virus (HSV) can lead to the disseminated HSV infection eczema herpeticatum, probably the most feared complication of AD (53). In addition, Malassezia yeast species colonize the skin of $90 \%$ of $\mathrm{AD}$ patients compared with $35 \%$ of healthy controls, especially the sebaceous areas of the face, scalp and upper body. Species associated with $\mathrm{AD}$ include Malassezia globosa, sympodialis, restricta, and furfur (54). Their role in $\mathrm{AD}$ exacerbations is controversial despite the fact that specific IgE antibodies towards Malassezia species can be found in $\mathrm{AD}$ patients but not in healthy controls (55). No evidence supports that antifungal treatments reducing Malassezia colonization would relieve $\mathrm{AD}$ in the long-term, although treatment periods with an antifungal agent have had some effect, especially on eczema in the sebaceous areas. Box 5 summarizes the main conclusions concerning the skin microbiome.

\section{Box 5 Main conclusions concerning the skin} microbiome.

- $\mathrm{AD}$ is associated with loss of diversity of the skin microbiome

- Staphylococcus aureus colonization is associated with increased disease severity

- When overt clinical infection, antibiotic treatment should be considered

\section{Treatment algorithm proposal}

Considering all the different treatment approaches in $\mathrm{AD}$, we aimed to develop a rationale and step by step approach according to its degree of severity and control- figures $\mathbf{2}$ and 3.

\section{Assessing disease severity}

Regarding disease severity, it must be determined by evaluating both objective signs (physician assessments of disease severity) and subjective symptoms (patient-reported symptoms and Quality of life outcomes). One of the most commonly used tools for assessing AD severity is SCORing Atopic Dermatitis (SCORAD); SCORAD attributes around $60 \%$ of the total score to the intensity of lesions, $20 \%$ to spread and $20 \%$ to subjective signs scored by the patient(56). A SCORAD $>50$ is regarded as severe, while SCORAD scores $<25$ are considered mild. Considering Quality of life, Dermatology Quality of life questionnaires (DLQI) and the Infants' Dermatology Quality of Life Index (IDQOL) are the QoL instruments most commonly used in $\mathrm{AD}$, taking into account the different disease domains, in particular signs and symptoms; sleep quality; work performance and social and emotional well-being; to quantify the different aspects of the individual burden of $\mathrm{AD}$ in a real-world setting.

\section{Assessing control}

In contrast with other allergic diseases such as asthma, no clear and globally accepted definition of control exists for AD. Langan et al (57) recently described a totally controlled week as one in which symptoms are well controlled every day. A well-controlled week was one in which increased symptoms have occurred or treatment has been applied for a period of 2 days or less and symptoms are controlled most of the time. In every clinical evaluation $\mathrm{AD}$ control should be addressed evaluating daytime and nocturnal symptoms, limitation of activities, need of rescue treatment and occurrence of flares (figure 2).

$\mathrm{AD}$ treatment should be based on a personalized cycle of assessment, adjustment of treatment, and review of the response. For each patient in addition to treatment of modifiable risk factors such as stress, controller medication can be adjusted up and down in a stepwise approach to achieve good symptom control and minimize risk of future exacerbations. The number of well controlled weeks will give the clinician a measure of disease control in a determined period of time. Once AD control has been maintained for 2-3 months treatment may be stepped down in order to find the patient minimum effective treatment. If a patient has persisting uncontrolled symptoms and/or exacerbations despite 2-3 months of controller treatment, the clinician should assess and correct some problems before considering any step up in treatment: poor adherence, 
Figure 2 - Algorithm proposal for Atopic Dermatitis management. Consider stepping up treatment, with or without overlapping, to attain total control. Adapted from Global Initiative for asthma available at https://ginasthma.org.

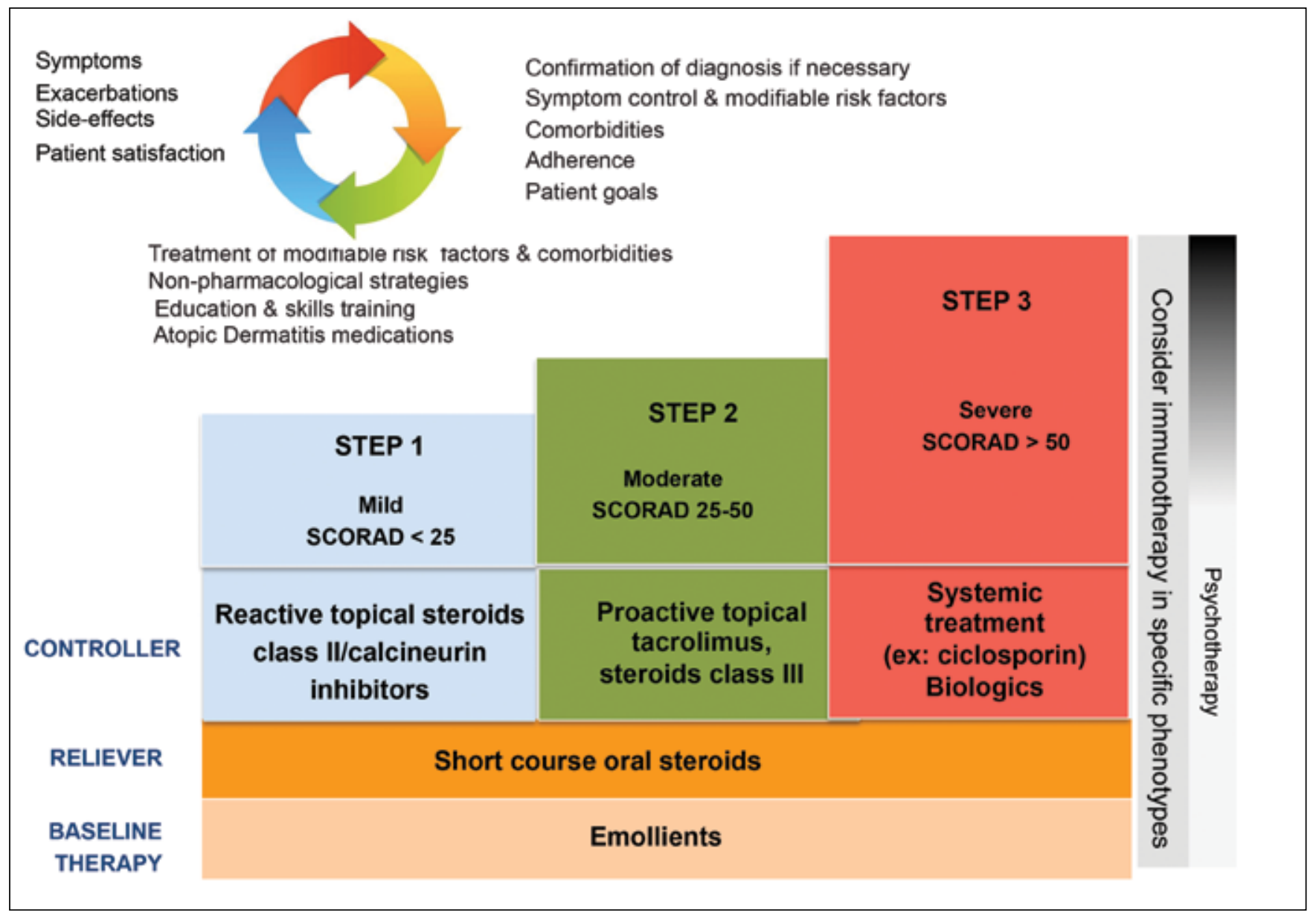

persistent exposure to home/work agents such as allergens, comorbidities that may contribute to poor quality of life and incorrect diagnosis.

\section{Key points regarding stepwise approach of $\mathrm{AD}$ treatment:}

\section{Mild Atopic dermatitis}

- When used on a daily basis, moisturizers with non-aqueous emollients, occlusive agents and humectants improve barrier function; reduce $\mathrm{AD}$ signs and symptoms, and the need for topical corticosteroids.

- Topical corticosteroids remain the first line treatment, reducing disease recurrence when used intermittently in patients with established disease.

Stepping up if $\mathrm{AD}$ remains uncontrolled despite good adherence:

- for patients with persistent symptoms and /or flares consider proactive therapy with topical tacrolimus or glucocorticosteroids class III;

- if disease control cannot be achieved with topical measures, when topical therapies fail or become unacceptable or impractical, systemic therapy is indicated.

Stepping down to find the minimum effective dose:

- consider step-down once AD control has been achieved and maintained for about 3 months, to find the lowest treatment that controls both symptoms and exacerbations;

- provide the patient with a written $\mathrm{AD}$ action plan, monitor closely and schedule a follow up visit in a 3-4 month period.

For all patients with $\mathrm{AD}$ :

- encourage adherence to emollients use, even when symptoms are infrequent;

- provide training in $\mathrm{AD}$ self-management to control symptoms and minimize risk of exacerbations. 
Figure 3 - Definition of Atopic Dermatitis total, well and uncontrolled weeks. A totally controlled week as one in which symptoms are well controlled every day. A well-controlled week was one in which increased symptoms have occurred or treatment has been applied for a period of 2 days or less and symptoms are controlled most of the time.

\begin{tabular}{|c|c|c|c|}
\hline Characteristics & $\begin{array}{l}\text { Total Controlled } \\
\text { week } \\
\text { (All of the following) }\end{array}$ & $\begin{array}{l}\text { Well controlled } \\
\text { week } \\
\text { (All of the following) }\end{array}$ & $\begin{array}{c}\text { Uncontrolled } \\
\text { week } \\
\text { (Any present) }\end{array}$ \\
\hline Daytime symptoms & None & $\leq 4$ times & Most of the days \\
\hline $\begin{array}{l}\text { Limitations of } \\
\text { activities }\end{array}$ & None & $\leq 4$ times & Most of the days \\
\hline $\begin{array}{l}\text { Nocturnal } \\
\text { symptoms / } \\
\text { awakening }\end{array}$ & None & $\leq 4$ times & Most of the days \\
\hline $\begin{array}{c}\text { Need for rescue / } \\
\text { "reliever" } \\
\text { treatment }\end{array}$ & None & $\leq 2$ days & $\geq 3$ days \\
\hline Flare* & None & None & One in any week \\
\hline
\end{tabular}

A "flare" of AD is defined as an episode resulting in behavior such as requiring an escalation of treatment (independently of the baseline grade of severity); or seeking additional medical advice

\section{Conclusions}

$\mathrm{AD}$ is a complex disease with host and environmental factors underlying its pathology. There are several different treatment approaches in $\mathrm{AD}$, such as emollients, topical steroids, calcineurin inhibitors, systemic general immunosupressors and monoclonal antibodies. Dupilumab is the only biologic currently approved for adolescents and adult patients with moderate-to-severe AD. Biologics targeting IgE/Th2 pathways may have its role in patients with overlapping $\mathrm{AD}$ and asthma.

We propose a new step by step approach aiming at maintaining disease control and improving quality of life.

\section{Conflict of interests}

Author Anabela Lopes declares collaborating and receiving fees from Novartis, Menarini and SANOFI through either participation in advisory boards or consultancy meetings or congress symposia. Author Anna Sokolova declares collaborating and receiving fees from Novartis, Astra Zeneca and Vitoria through either participation in advisory boards or consultancy meetings or congress symposia. Author Carmo Abreu declares collaborating and receiving fees from Novartis through either partici- pation in advisory boards or consultancy meetings or congress symposia. Author Cristina Lopes declares collaborating and receiving fees from Astra-Zeneca, Novartis, Menarini, TEVA and SANOFI, through either participation in advisory boards or consultancy meetings or congress symposia.

\section{Acknowledgments}

Funding for this paper was provided by Novartis Portugal. Funding was used to access all necessary scientific bibliography and cover meeting expenses. Novartis Portugal had no role in the collection, analysis and interpretation of data, in the writing of the paper or in the decision to submit the paper for publication.

\section{References}

1. Flohr C, Mann J. New insights into the epidemiology of childhood atopic dermatitis. Allergy. 2014;69(1):3-16.

2. Henderson J, Northstone K, Lee SP, Liao H, Zhao Y, Pembrey M, et al. The burden of disease associated with filaggrin mutations: a population-based, longitudinal birth cohort study. The Journal of allergy and clinical immunology. 2008;121(4):872-7 e9.

3. Haahtela T, Holgate S, Pawankar R, Akdis CA, Benjaponpitak S, Caraballo L, et al. The biodiversity hypothesis and allergic disease: 
world allergy organization position statement. The World Allergy Organization journal. 2013;6(1):3.

4. Ring J, Mohrenschlager M, Weidinger S. Molecular genetics of atopic eczema. Chemical immunology and allergy. 2012;96:24-9.

5. Kim BE, Leung DY, Boguniewicz M, Howell MD. Loricrin and involucrin expression is down-regulated by Th2 cytokines through STAT-6. Clinical immunology (Orlando, Fla). 2008;126(3):332-7.

6. Darsow U, Wollenberg A, Simon D, Taieb A, Werfel T, Oranje A, et al. ETFAD/EADV eczema task force 2009 position paper on diagnosis and treatment of atopic dermatitis. Journal of the European Academy of Dermatology and Venereology : JEADV. 2010;24(3):317-28.

7. Tollefson MM, Bruckner AL. Atopic dermatitis: skin-directed management. Pediatrics. 2014;134(6):e1735-44.

8. Eichenfield LF, Tom WL, Berger TG, Krol A, Paller AS, Schwarzenberger $\mathrm{K}$, et al. Guidelines of care for the management of atopic dermatitis: section 2. Management and treatment of atopic dermatitis with topical therapies. Journal of the American Academy of Dermatology. 2014;71(1):116-32.

9. Wollenberg A, Barbarot S, Bieber T, Christen-Zaech S, Deleuran M, Fink-Wagner A, et al. Consensus-based European guidelines for treatment of atopic eczema (atopic dermatitis) in adults and children: part I. Journal of the European Academy of Dermatology and Venereology : JEADV. 2018;32(5):657-82.

10. Lee HJ, Lee SH. Epidermal permeability barrier defects and barrier repair therapy in atopic dermatitis. Allergy, asthma $\&$ immunology research. 2014;6(4):276-87.

11. Resumo Caracteristicas do Medicamento Dupixent ( 2018 [Available from: https://ec.europa.eu/health/documents/community-register/2017/20170926138667/anx_138667_pt.pdf.

12. van Zuuren EJ, Fedorowicz Z, Arents BWM. Emollients and moisturisers for eczema: abridged Cochrane systematic review including GRADE assessments. The British journal of dermatology. 2017.

13. Boralevi F, Saint Aroman M, Delarue A, Raudsepp H, Kaszuba A, Bylaite $\mathrm{M}$, et al. Long-term emollient therapy improves xerosis in children with atopic dermatitis. Journal of the European Academy of Dermatology and Venereology : JEADV. 2014;28(11):1456-62.

14. Torres T, Ferreira EO, Goncalo M, Mendes-Bastos P, Selores M, Filipe P. Update on Atopic Dermatitis. Acta Med Port. 2019;32(9):606-13.

15. Halling-Overgaard AS, Kezic S, Jakasa I, Engebretsen KA, Maibach $\mathrm{H}$, Thyssen JP. Skin absorption through atopic dermatitis skin: a systematic review. The British journal of dermatology. 2017;177(1):84-106.

16. Broeders JA, Ahmed Ali U, Fischer G. Systematic review and meta-analysis of randomized clinical trials (RCTs) comparing topical calcineurin inhibitors with topical corticosteroids for atopic dermatitis: A 15-year experience. Journal of the American Academy of Dermatology. 2016;75(2):410-9 e3.

17. Mollanazar NK, Smith PK, Yosipovitch G. Mediators of Chronic Pruritus in Atopic Dermatitis: Getting the Itch Out? Clinical reviews in allergy \& immunology. 2016;51(3):263-92.

18. Sidbury R, Davis DM, Cohen DE, Cordoro KM, Berger TG, Bergman JN, et al. Guidelines of care for the management of atopic dermatitis: section 3. Management and treatment with phototherapy and systemic agents. Journal of the American Academy of Dermatology. 2014;71(2):327-49.

19. Simon D, Bieber T. Systemic therapy for atopic dermatitis. Allergy. $2014 ; 69(1): 46-55$.
20. Patrizi A, Raone B, Ravaioli GM. Management of atopic dermatitis: safety and efficacy of phototherapy. Clinical, cosmetic and investigational dermatology. 2015;8:511-20.

21. Wollenberg A, Barbarot S, Bieber T, Christen-Zaech S, Deleuran M, Fink-Wagner A, et al. Consensus-based European guidelines for treatment of atopic eczema (atopic dermatitis) in adults and children: part II. Journal of the European Academy of Dermatology and Venereology : JEADV. 2018;32(6):850-78.

22. Wollenberg A, Barbarot S, Bieber T, Christen-Zaech S, Deleuran $\mathrm{M}$, Fink-Wagner A, et al. Consensus-based European guidelines for treatment of atopic eczema (atopic dermatitis) in adults and children: part II. Journal of the European Academy of Dermatology and Venereology : JEADV. 2018;32(6):850-78.

23. Koppelstaetter C, Kern G, Leierer G, Mair SM, Mayer G, Leierer J. Effect of cyclosporine, tacrolimus and sirolimus on cellular senescence in renal epithelial cells. Toxicology in vitro : an international journal published in association with BIBRA. 2018;48:86-92.

24. Megna M, Napolitano M, Patruno C, Villani A, Balato A, Monfrecola G, et al. Systemic Treatment of Adult Atopic Dermatitis: A Review. Dermatology and therapy. 2017;7(1):1-23.

25. Holm JG, Agner T, Sand C, Thomsen SF. Omalizumab for atopic dermatitis: case series and a systematic review of the literature. International journal of dermatology. 2017;56(1):18-26.

26. Summary of Product Characteristics Omalizumab. Approved by EMA 2009. Last updated 2016.

27. Thaiwat S, Sangasapaviliya A. Omalizumab treatment in severe adult atopic dermatitis. Asian Pacific journal of allergy and immunology. 2011;29(4):357-60.

28. Andreae DA, Wang J. Immunologic effects of omalizumab in children with severe refractory atopic dermatitis: a randomized, placebo-controlled clinical trial. Pediatrics. 2014;134 Suppl 3:S160.

29. Chan S, Cornelius V, Cro S, Harper JI, Lack G. Treatment Effect of Omalizumab on Severe Pediatric Atopic Dermatitis: The ADAPT Randomized Clinical Trial. JAMA Pediatr. 2019.

30. Hotze M, Baurecht H, Rodriguez E, Chapman-Rothe N, Ollert $\mathrm{M}$, Folster-Holst R, et al. Increased efficacy of omalizumab in atopic dermatitis patients with wild-type filaggrin status and higher serum levels of phosphatidylcholines. Allergy. 2014;69(1):132-5.

31. Wang HH, Li YC, Huang YC. Efficacy of omalizumab in patients with atopic dermatitis: A systematic review and meta-analysis. The Journal of allergy and clinical immunology. 2016;138(6):1719-22 e1.

32. Velling P, Skowasch D, Pabst S, Jansen E, Tuleta I, Grohe C. Improvement of quality of life in patients with concomitant allergic asthma and atopic dermatitis: one year follow-up of omalizumab therapy. European journal of medical research. 2011;16(9):40710.

33. Beck LA, Thaci D, Hamilton JD, Graham NM, Bieber T, Rocklin R, et al. Dupilumab treatment in adults with moderate-to-severe atopic dermatitis. The New England journal of medicine. 2014;371(2):130-9.

34. Simpson EL, Bieber T, Guttman-Yassky E, Beck LA, Blauvelt A, Cork MJ, et al. Two Phase 3 Trials of Dupilumab versus Placebo in Atopic Dermatitis. The New England journal of medicine. 2016;375(24):2335-48.

35. 2017 SoPCDAbE. <RCM Dupilumab.pdf>. Last Updated 2017.

36. Simpson EL, Paller AS, Siegfried EC, Boguniewicz M, Sher L, Gooderham MJ, et al. Efficacy and Safety of Dupilumab in Adolescents With Uncontrolled Moderate to Severe Atopic Dermatitis: A Phase 3 Randomized Clinical Trial. JAMA Dermatol. 2019. 
37. Corren J, Castro M, O'Riordan T, Hanania NA, Pavord ID, Quirce S, et al. Dupilumab Efficacy in Patients with Uncontrolled, Moderate-to-Severe Allergic Asthma. J Allergy Clin Immunol Pract. 2019.

38. Bachert C, Han JK, Desrosiers M, Hellings PW, Amin N, Lee $\mathrm{SE}$, et al. Efficacy and safety of dupilumab in patients with severe chronic rhinosinusitis with nasal polyps (LIBERTY NP SINUS-24 and LIBERTY NP SINUS-52): results from two multicentre, randomised, double-blind, placebo-controlled, parallel-group phase 3 trials. Lancet (London, England). 2019;394(10209):1638-50.

39. Chida Y, Steptoe A, Hirakawa N, Sudo N, Kubo C. The effects of psychological intervention on atopic dermatitis. A systematic review and meta-analysis. International archives of allergy and immunology. 2007;144(1):1-9.

40. Walker IC. Causation of eczema, urticaria, and angioneurotic edema by proteins other than those derived from foods. Jama. 1918;70:897-900.

41. Knaysi G, Smith AR, Wilson JM, Wisniewski JA. The Skin as a Route of Allergen Exposure: Part II. Allergens and Role of the Microbiome and Environmental Exposures. Current allergy and asthma reports. 2017;17(1):7.

42. Nakamura T, Hirasawa Y, Takai T, Mitsuishi K, Okuda M, Kato T, et al. Reduction of skin barrier function by proteolytic activity of a recombinant house dust mite major allergen Der $f 1$. The Journal of investigative dermatology. 2006;126(12):2719-23.

43. Capristo C, Romei I, Boner AL. Environmental prevention in atopic eczema dermatitis syndrome (AEDS) and asthma: avoidance of indoor allergens. Allergy. 2004;59 Suppl 78:53-60.

44. Nankervis H, Pynn EV, Boyle RJ, Rushton L, Williams HC, Hewson DM, et al. House dust mite reduction and avoidance measures for treating eczema. The Cochrane database of systematic reviews. 2015;1:CD008426.

45. Werfel T, Breuer K, Rueff F, Przybilla B, Worm M, Grewe M, et al. Usefulness of specific immunotherapy in patients with atopic dermatitis and allergic sensitization to house dust mites: a multi-centre, randomized, dose-response study. Allergy. 2006;61(2):202-5.

46. Wassmann A, Werfel T. Atopic eczema and food allergy. Chemical immunology and allergy. 2015;101:181-90.

47. Capone KA, Dowd SE, Stamatas GN, Nikolovski J. Diversity of the human skin microbiome early in life. The Journal of investigative dermatology. 2011;131(10):2026-32.
48. Soares J, Lopes C, Tavaria F, Delgado L, Pintado M. A diversity profile from the staphylococcal community on atopic dermatitis skin: a molecular approach. J Appl Microbiol. 2013;115(6):1411-9.

49. Bath-Hextall FJ, Birnie AJ, Ravenscroft JC, Williams HC. Interventions to reduce Staphylococcus aureus in the management of atopic eczema: an updated Cochrane review. The British journal of dermatology. 2010;163(1):12-26.

50. Gong JQ, Lin L, Lin T, Hao F, Zeng FQ, Bi ZG, et al. Skin colonization by Staphylococcus aureus in patients with eczema and atopic dermatitis and relevant combined topical therapy: a double-blind multicentre randomized controlled trial. The British journal of dermatology. 2006;155(4):680-7.

51. Lopes C, Silva D, Delgado L, Correia O, Moreira A. Functional textiles for atopic dermatitis: a systematic review and meta-analysis. Pediatric allergy and immunology : official publication of the European Society of Pediatric Allergy and Immunology. 2013;24(6):603-13.

52. Lopes C, Soares J, Tavaria F, Duarte A, Correia O, Sokhatska O, et al. Chitosan Coated Textiles May Improve Atopic Dermatitis Severity by Modulating Skin Staphylococcal Profile: A Randomized Controlled Trial. PloS one. 2015;10(11):e0142844.

53. Wetzel S, Wollenberg A. [Eczema herpeticatum]. Der Hautarzt; Zeitschrift fur Dermatologie, Venerologie, und verwandte Gebiete. 2004;55(7):646-52.

54. Zargari A, Midgley G, Bäck O, Johansson SG, Scheynius A. IgE-reactivity to seven Malassezia species. Allergy. 2003;58(4):306-11.

55. Roll A, Cozzio A, Fischer B, Schmid-Grendelmeier P. Microbial colonization and atopic dermatitis. Current opinion in allergy and clinical immunology. 2004;4(5):373-8.

56. Severity scoring of atopic dermatitis: the SCORAD index. Consensus Report of the European Task Force on Atopic Dermatitis. Dermatology (Basel, Switzerland). 1993;186(1):23-31.

57. Langan SM, Stuart B, Bradshaw L, Schmitt J, Williams HC, Thomas KS. Measuring long-term disease control in patients with atopic dermatitis: A validation study of well-controlled weeks. The Journal of allergy and clinical immunology. 2017.

58. Anderson GP. Endotyping asthma: new insights into key pathogenic mechanisms in a complex, heterogeneous disease. Lancet (London, England). 2008;372(9643):1107-19. 